\title{
Culture technique et enseignement professionnel dans les écoles d'horlogerie suisses (1850-1920)
}

From Excellence to Utilitarianism. Technical Culture and Professional Education in Swiss Clock-Making Schools (1850-1920)

Vom Streben nach Exzellenz zum Nützlichkeitsprinzip: Fachspezfische Kultur und Berufsausbildung in Schweizer Uhrmacherschulen zwischen 1850 und 1920 De la excelencia al utilitarismo. Cultura técnica y enseñanza profesional en las escuelas de relojería suizas (1850-1920)

\section{Pierre-Yves Donzé}

\section{OpenEdition Journals}

\section{Édition électronique}

URL : https://journals.openedition.org/histoire-education/1841

DOI : 10.4000/histoire-education.1841

ISSN : 2102-5452

\section{Éditeur}

ENS Éditions

\section{Édition imprimée}

Date de publication : 1 juillet 2008

Pagination : $5-28$

ISBN : 978-2-7342-1124-2

ISSN : 0221-6280

\section{Référence électronique}

Pierre-Yves Donzé, «Culture technique et enseignement professionnel dans les écoles d'horlogerie suisses (1850-1920) », Histoire de l'éducation [En ligne], 119 | 2008, mis en ligne le 01 janvier 2013, consulté le 20 mai 2021. URL : http://journals.openedition.org/histoire-education/1841 ; DOI : https:// doi.org/10.4000/histoire-education. 1841 


\title{
De l'excellence à l'utilitarisme \\ Culture technique et enseignement professionnel dans les écoles d'horlogerie suisses (1850-1920)
}

\author{
Pierre-Yves DONZÉ
}

L'histoire de l'éducation technique est un champ de recherches relativement récent ${ }^{1}$. En Suisse, il n'a donné lieu qu'à un petit nombre de travaux universitaires qui, mis à part l'ouvrage de Jean-Pierre Tabin ${ }^{2}$, se limitent généralement à un espace déterminé (Genève, Vaud) ${ }^{3}$ ou à un secteur d'activité particulier, comme l'horlogerie ${ }^{4}$. Les années 1850-1920 apparaissent comme un moment déterminant dans la mise en place des structures au sein desquelles sont transmis des savoirfaire attachés à l'exercice des professions. Or, la définition des cultures techniques enseignées aux nouvelles générations pose alors un certain nombre de problèmes, tels qu'il s'en rencontre dans les pays en cours d'industrialisation.

De manière générale, en Suisse, l'apprentissage technique est dominé au $\mathrm{XIX}^{\mathrm{e}}$ siècle par deux grands courants : l'un, de tendance conservatrice, vise à

1 Pour une synthèse des approches historiographiques actuelles, voir Thérèse Charmasson (dir.), Formation au travail, enseignement technique et apprentissage, Paris, CTHS, 2005.

2 Jean-Pierre Tabin, Formation professionnelle en Suisse. Histoire et actualité, Lausanne, Réalités sociales, 1989.

3 Liliane Fazan, La politique de l'apprentissage à Genève de 1892 à 1930. De la première loi genevoise à la première loi fédérale sur la formation professionnelle, mémoire de licence, Université de Genève, 1981, et Acacio Calisto, Apprentissage : vers la formation en série de main-d'œuvre qualifiée. Politique de formation professionnelle dans le canton de Vaud, 1896-1936, mémoire de licence, Université de Lausanne, 2001.

4 Estelle Fallet, Alain Cortat, Apprendre l'horlogerie dans les Montagnes neuchâteloises, 1740-1810, La Chaux-de-Fonds, Institut L'homme et le temps, 2001, et Apprendre, créer, transmettre. La formation des horlogers, passé et avenir, La Chaux-de-Fonds, Institut L'homme et le temps, 1999. 
réhabiliter les métiers traditionnels mis à mal par l'industrialisation ; l'autre, de nature utilitariste, a pour objectif la formation d'une main-d'œuvre qualifiée nécessaire aux entreprises industrielles. D'un côté, en effet, il faut compter avec une réaction contre le libéralisme économique, qui a aboli les corporations. Ce courant, qui milite pour la réhabilitation des métiers traditionnels au travers de l'apprentissage ${ }^{5}$, est très en vogue parmi les petits entrepreneurs et les artisans. Ceux-ci se regroupent en 1879 au sein d'une association, l'Union suisse des arts et métiers (USAM), qui entreprend d'intervenir dans le champ politique, et sont accompagnés dans leurs revendications par plusieurs philanthropes, pédagogues et inspecteurs des fabriques, choqués par la situation socialement difficile et non réglementée dans laquelle se réalise la formation professionnelle. L'Assemblée fédérale, saisie du problème de l'apprentissage en 1882, entreprend une enquête industrielle qui révèle l'état déplorable des conditions de vie des apprentis. Sur la demande de l'USAM, la Confédération accepte alors d'accorder des subsides aux écoles professionnelles (1884). C'est pourtant au niveau cantonal que s'observe une première réglementation de l'apprentissage, qui porte principalement sur la généralisation des contrats et l'instauration de commissions de surveillance : des lois sont adoptées dans les cantons de Neuchâtel (1890), Genève (1892), Fribourg (1895), Berne (1905), Zurich (1906), etc. Ce n'est qu'en 1930 que la Confédération adoptera une loi fédérale sur les apprentissages, unifiant ainsi les diverses législations cantonales. Cette prise de conscience politique et l'engagement financier des collectivités publiques qui en résulte permettent un premier essor des écoles professionnelles : tandis qu'en 1884, la Confédération accorde 40000 francs à 43 établissements, ce sont 318 écoles qui sont soutenues en 1904, recevant au total plus d'un million de francs ${ }^{6}$.

D’autre part, il faut prendre en considération l'action déployée par les milieux industriels. S'ils se désintéressent passablement de la réhabilitation des métiers traditionnels, en raison de leurs besoins en main-d'œuvre peu qualifiée et bon marché, ils n'en demeurent pas moins favorables à l'éducation technique de leurs employés. Mais ce que veulent les industriels, c'est une formation pratique, directement applicable dans le travail quotidien. On ne cherche pas à transmettre une culture technique ancestrale, mais de nouvelles connaissances nécessaires à l'utilisation de machines sans cesse perfectionnées. Ainsi que l'affirme Philippe

\footnotetext{
5 Muriel Surdez, Diplômes et nation. La constitution d'un espace suisse des professions avocate et artisanales (1880-1930), Berne, Peter Lang, 2005. 
Marchand, il s'agit de mettre sur pied " un enseignement professionnel apte à fournir les sous-officiers de l'armée industrielle $"^{7}$. Le patronat industriel fonde ainsi ses propres écoles ou intervient, sous des formes diverses (financement, présence dans les commissions administratives, supervision de la formation pratique, etc.), dans les établissements existants.

La conciliation entre ces deux tendances n'est pas toujours facile, en particulier durant la seconde partie du XIX ${ }^{\mathrm{e}}$ siècle, qui voit s'affronter les défenseurs d'une culture technique fondée sur la tradition d'excellence et les partisans de savoir-faire modernisés, utiles à des entreprises en pleine mutation technologique. L'horlogerie suisse apparaît dans cette perspective comme un excellent exemple à étudier. La dizaine d'écoles d'horlogerie que compte le pays sont, en effet, fondées vers le milieu du XIX ${ }^{\mathrm{e}}$ siècle par des fabricants désireux de former une élite de techniciens maitrisant la culture technique de leur branche dans son ensemble. Dans les années 1870-1900, ces établissements sont le lieu d'intenses débats sur la question de la modernisation de l'enseignement dispensé, ainsi que sur son rôle dans un secteur industriel en voie de mécaniser son mode de production ${ }^{8}$.

$\mathrm{Si}$, de manière générale, la transformation des structures traditionnelles de l'apprentissage conformément à un modèle utilitariste soulève des problèmes que l'on rencontre ailleurs en Europe à la fin du XIX siècle, l'horlogerie suisse présente des caractéristiques singulières, qui en font un cas tout à fait particulier ${ }^{9}$. D'abord, cette industrie n'a pas connu de corporations, sauf dans la ville de Genève sous l'Ancien Régime. L'horlogerie suisse s'est développée depuis la seconde partie du $\mathrm{XVII}{ }^{\mathrm{e}}$ siècle en dehors des villes, dans l'arc jurassien, et c'est précisément l'absence de corporations qui a permis l'essor de cette branche sous la forme d'un système de production particulièrement flexible. L'apprentissage du métier d'horloger se réalise ainsi essentiellement selon des modalités variables, définies par des contrats privés ${ }^{10}$. La création des écoles d'horlogerie au milieu du XIX siècle ne s'explique donc pas par une volonté de renforcer un modèle corporatif en crise : elle apparaît

7 Philippe Marchand, "Les écoles pratiques de commerce et d'industrie dans le Nord de la France, 1892-1940. Jalons pour une histoire nationale de l'enseignement technique et professionnel moyen ", in Thérèse Charmasson (dir.), op. cit., p. 44.

8 Sur l'évolution économique et technologique de l'industrie horlogère suisse durant ces années, voir Patrick Linder, Organisation et technologie : un système industriel en mutation. L'horlogerie à Saint-Imier, 1865-1918, mémoire de licence, Université de Neuchâtel, 2006.

9 Sur l'histoire de l'horlogerie suisse, voir David S. Landes, L'heure qu'il est. Les horloges, la mesure du temps et la formation du monde moderne, Paris, Gallimard, 1987, et Catherine Cardinal (dir.), L'homme et le temps en Suisse, 1291-1991, La Chaux-de-Fonds, Institut l'homme et le temps, 1991,

Estelle Fallet et Alain Cortat, op. cit. 
plutôt comme un geste réactionnaire à l'encontre d'un système d'apprentissage utilitariste. Ensuite, l'industrie horlogère suisse se caractérise par l'absence de grandes entreprises et par son organisation sous forme de district industriel. Le recensement fédéral des entreprises de 1905 en dénombre seulement sept qui emploient plus de 500 personnes en usine ; la plus grande de ces entreprises, Langendorf SA, n'a qu'un effectif de 1098 employés ${ }^{11}$. En 1901, on compte 663 entreprises horlogères, et leur nombre moyen d'employés s'élève à moins de $40^{12}$. L'absence de grandes entreprises explique largement que la création d'écoles d'horlogerie résulte d'initiatives collectives du petit patronat industriel. À de rares exceptions près, comme celle de la maison Omega, les fabricants d'horlogerie n'ont pas les moyens financiers de mettre sur pied leur propre centre de formation. Ils s'associent alors pour créer des établissements d'apprentissage et obtenir le soutien des collectivités publiques. L'absence de corporation et l'organisation sous forme de district industriel font ainsi de l'horlogerie suisse un exemple qui permet d'appréhender l'institutionnalisation de la formation technique sans recourir à l'explication évolutionniste, puisque ce ne sont ni le désir d'un retour au modèle corporatif ni l'essor de la grande entreprise industrielle qui forment le cadre dans lequel elle s'inscrit.

\section{I - La naissance des écoles d'horlogerie (1850-1880)}

L'horlogerie connaît un formidable essor au cours du XVIII siècle dans l'ensemble de l'arc jurassien. Le développement de ce secteur d'activité se réalise dans le cadre de l'établissage ${ }^{13}$ (domestic system), caractérisé par la fragmentation de la production entre des dizaines d'unités de production. Dans ce contexte, la forte division du travail existant dans l'horlogerie débouche, dès le milieu du XVIII siècle, sur une tendance à ne faire que des apprentissages de courte durée, portant sur une partie seulement du processus de production ${ }^{14}$. L'absence de corporations

11 Marius Fallet-Scheurer, Le travail à domicile dans l'horlogerie suisse et ses industries annexes, Berne, 1912 , p. 314.

12 Feuille fédérale, 1931, p. 193.

13 Établissage : mode de fabrication de la montre et/ou du mouvement consistant à en assembler les divers éléments constitutifs. Il comporte généralement les opérations suivantes : réception, contrôle et stockage des ébauches, des parties réglantes, ainsi que des autres fournitures du mouvement et de l'habillement ; remontage ; réglage ; posage du cadran et des aiguilles ; emboîtage ; contrôle final, avant emballage et expédition.

Estelle Fallet et Alain Cortat, op. cit. 
- à l'exception notable de la ville de Genève - facilite par ailleurs la flexibilité de ce mode de formation et son adaptation aux besoins fluctuants de l'économie.

Ce système de formation est remis en cause, dans la première partie du $\mathrm{XIX}^{\mathrm{e}}$ siècle, par une élite de fabricants qui juge dangereuse cette tendance à diviser et limiter les connaissances techniques. Craignant à terme la perte de leur culture technique, ils promeuvent la mise sur pied d'écoles formant des horlogers qui maitrisent l'ensemble du processus de fabrication de la montre, en proposant un apprentissage sur trois ans alliant enseignement théorique (physique, astronomie, dessin, mathématiques, etc.) et travail pratique en atelier. Ces établissements doivent permettre la formation d'une nouvelle élite horlogère, destinée à prendre en main la direction des comptoirs et des ateliers. C'est à Genève que le mouvement est lancé, avec l'ouverture de la première école d'horlogerie de Suisse en $1824^{15}$. L'abolition, pendant la période révolutionnaire, du régime corporatif auquel était soumise la production horlogère de Genève sous l'Ancien Régime nécessite, en effet, la réorganisation de la formation professionnelle des horlogers sous une nouvelle forme institutionnelle. Cette école s'inscrit dans la continuité du XVIII ${ }^{\mathrm{e}}$ siècle genevois : elle a pour but d'alimenter les ateliers de la ville en horlogers complets, comme le faisait avant 1798 la corporation horlogère.

L'exemple genevois est repris ailleurs en Suisse, d'abord à La Chaux-de-Fonds, où une école s'ouvre en 1865 après plusieurs années de débats, puis dans le reste de l'arc jurassien : à Saint-Imier (1866), au Locle (1868), à Neuchâtel (1871), à Bienne (1873) et à Fleurier (1875). Malgré ce décalage chronologique entre Genève et le reste de l'arc jurassien, qui s'explique par l'absence de corporations en dehors de la cité lémanique, l'idéologie qui sous-tend la création de ces établissements est similaire : on cherche à promouvoir l'excellence technique, ainsi que l'illustre l'exemple de l'École d'horlogerie de La Chaux-de-Fonds.

\section{1 - L'exemple de l'École d'horlogerie de La Chaux-de-Fonds (1865-1880)}

L'École d'horlogerie de La Chaux-de-Fonds est ouverte en 1865 par l'élite des établisseurs de la ville, qui organise une école dans le but de transmettre des connaissances complètes à un petit nombre d'horlogers. Le besoin d'un tel établissement s'était fait sentir dès le début du XIX ${ }^{\mathrm{e}}$ siècle et de premières tentatives avaient été faites dans la seconde partie des années 1820, mais elles n'avaient pas abouti, pour des raisons matérielles. C'est en fin de compte un legs de biens fonciers à la municipalité qui s'avère décisif, parce que résolvant en grande partie 
la question financière. Les autorités communales travaillent alors à l'organisation de l'établissement, qu'elles créent sous leur autorité en 1865. Lors de son ouverture, l'école propose un apprentissage en trois ans. Durant leur formation, les élèves passent par trois classes (ébauches ${ }^{16}$, finissages ${ }^{17}$, pignons ; échappements ; remontage et repassage), qui doivent leur donner des connaissances étendues sur l'ensemble des techniques entrant dans le processus de fabrication de la montre. Il s'agit donc bien d'assurer la permanence d'une élite horlogère et de lutter contre ce que la commission administrative de l'école appelle en 1874 " la rareté croissante de bons ouvriers, des horlogers vraiment dignes de ce nom " ${ }^{18}$. Ce caractère élitiste est d'ailleurs entretenu par un écolage annuel qui se monte à près de 500 francs et qui assure une part importante du fonctionnement de l'école : pour l'exercice 1865-1866, le montant des écolages se monte à 7567 francs et représente 60,7 \% des recettes ${ }^{19}$.

L'École est elle-même dirigée par d'ardents défenseurs de l'excellence technique horlogère. Son premier comité de douze membres, nommé en 1865, fait une large place aux représentants des anciennes familles de fabricants, réticents face à l'introduction de machines dans les ateliers. Toutefois, l'ambition d'excellence technique des fondateurs de l'école ne correspond pas aux réels besoins de la population horlogère. Malgré un succès certain de l'établissement, qui voit le nombre de ses élèves passer de 15 en 1865 à 35 en 1870, la durée d'apprentissage complet de trois ans est très peu suivie. Selon un rapport établi par le directeur, parmi les 140 élèves ayant fréquenté l'École entre 1865 et 1874, seulement 14 (10\%) y ont étudié durant trois ans, tandis que 50 ont suivi les cours pendant moins d'un an (35,7\%), 51 pendant moins de deux ans (36,4\%) et 39 pendant moins de trois ans $(27,9 \%)^{20}$. Cette inadéquation avec les besoins du marché met l'école sous le feu des critiques. Dans une lettre envoyée en décembre 1874 au conseil communal, la commission administrative défend sa position et dénonce " la fâcheuse tendance de l'époque actuelle qui cherche à émanciper le jeune homme le plus tôt possible. Beaucoup de parents, pour alléger leurs charges, beaucoup de jeunes gens, pour être plus vite indépendants, trouvent que les cours de l'École d'horlogerie sont trop

16 Ébauche : Ensemble de pièces non assemblées du mouvement (platine, ponts, rouage, mécanisme de remontage et de mise à l'heure, raquette de réglage) et commercialisées sous cette forme.

17 Finissage : Dernier travail pour assembler et faire fonctionner les diverses parties de la montre.

18 Archives municipales de La Chaux-de-Fonds (ACHX), lettre de la commission de l'École au conseil municipal, 10 décembre 1874.

19 Samuel Guye, Histoire de l'École d'horlogerie de La Chaux-de-Fonds, La Chaux-de-Fonds, 1965, p. 30.

20 Ibid., p. 43. 
longs et l'on préfère un apprentissage incomplet où l'on supplée au raisonnement par la routine, afin d'être plus rapidement en état de se suffire à soi-même " ${ }^{21}$. La commission conclut sa missive en déclarant que " la mission de l'École d'horlogerie est de rester une pépinière d'horlogers sérieux et non de suivre la tendance de l'époque ainsi que certaines personnes paraissent le désirer $" 22$.

\section{2 - Le difficile retour de Philadelphie (1876-1877)}

Lorsqu'il revient de l'Exposition universelle de Philadelphie, en 1876, l'ingénieur Jacques David, directeur technique des Longines, à Saint-Imier, est bien décidé à moderniser l'horlogerie suisse. Lors de son voyage aux États-Unis, il a visité les principales fabriques horlogères de ce pays (Waltham Watch et Elgin Watch) ${ }^{23}$. Organisées comme des entreprises industrielles, celles-ci produisent des montres extrêmement bon marché grâce à l'usage de machines et à la standardisation des produits, ce qui remet en question la domination exercée jusque-là par l'horlogerie suisse sur le marché américain, son principal débouché.

Jacques David veut adopter le modèle américain dans les fabriques horlogères suisses et développe dès 1876 une politique très active en faveur de ce projet, politique au sein de laquelle la formation professionnelle occupe une place de choix. Dans le rapport qu'il présente à la Société intercantonale des industries du Jura à son retour de Philadelphie, il accorde une place particulière aux écoles dans la réorganisation de l'horlogerie suisse. Il écrit en conclusion : "Les écoles d'horlogerie et les écoles de dessin destinées aux apprentis doivent être développées, l'accès doit en être rendu de plus en plus facile à tous et leur influence sera pour beaucoup dans les progrès que notre industrie doit étudier dès maintenant. Il est nécessaire pour cela qu'on fasse connaître aux élèves de ces écoles les outils et les machines en usage dans les établissements perfectionnés dont nous venons de parler, et qu'on leur donne quelques notions de ces nouveaux procédés de fabrication $"^{24}$.

Il est, par ailleurs, membre de la commission de l'École d'horlogerie de Saint-Imier, fondée en 1866, et s'y impose comme l'une des personnalités de l'établissement jusqu'à son décès en 1912. C'est sous son impulsion qu'une réunion

21 ACHX, lettre de la commission de l'École au conseil municipal, 10 décembre 1874.

22 Id.

23 Donald R. Hoke, Ingenious Yankees. The Rise of the American System of Manufacturers in the Private Sector, New York, Columbia University Press, 1990.

24 Jacques David, Rapport à la Société intercantonale des industries du Jura sur la fabrication de l'horlogerie aux États-Unis, 1876, fac-similé, 1992, p. 106. 
des directeurs des écoles d'horlogerie de Suisse a lieu à Neuchâtel, en 1877, pour débattre de l'éventualité " d'apporter une modification dans l'enseignement théorique et pratique donné actuellement dans nos écoles [...] ensuite de la concurrence [des] grands établissements américains travaillant par des procédés mécaniques " ${ }^{25}$. En clair, il s'agit de réorganiser et d'unifier la formation des horlogers en Suisse, en développant les connaissances dans le domaine mécanique et en introduisant des machines dans les ateliers. Aucune décision n'est prise durant cette réunion, qui révèle surtout des positions tranchées quant à l'adoption du modèle américain.

Les écoles de Genève, de La Chaux-de-Fonds et du Locle se montrent les plus réticentes. Pour elles, seule la promotion d'une horlogerie de qualité, produite de manière artisanale, est à même de combattre la concurrence étrangère. Pour ce faire, il est nécessaire de disposer d'écoles promouvant l'excellence technique d'un savoir-faire traditionnel. Le directeur de l'École d'horlogerie de La Chauxde-Fonds, Schouffelberger, déclare à cette occasion que la commission de son établissement " a été unanime en déclarant qu'il n'y a pas de grands changements à apporter dans l'enseignement horloger $"{ }^{26}$. Il s'agit, selon eux, de combattre la concurrence américaine grâce à la " bonne horlogerie " ${ }^{27}$. Les administrateurs de l'École d'horlogerie de Bienne, absents de la réunion de Neuchâtel, se montrent, eux aussi, peu favorables au projet de Jacques David, écrivant dans leur rapport d'activité pour l'année 1876-1877 que "l'horlogerie, étant un art, doit être guidée par la science ${ }^{28}$. C'est donc seul, dans le cadre de l'établissement de Saint-Imier, que Jacques David met en œuvre son projet modernisateur.

\section{II - Un établissement pionnier : l'École d'horlogerie de Saint-Imier (1866-1914)}

La création et le développement de l'École d'horlogerie de Saint-Imier sont intimement liés à la principale fabrique d'horlogerie de la localité, la fabrique des

\footnotetext{
25 Mémoires d'Ici, Saint-Imier (MDI), fonds École d'horlogerie de Saint-Imier, rapport de la réunion des directeurs des écoles d'horlogerie de Suisse romande, 17 mai 1877.

Rapport d'activité de l'École d'horlogerie de Bienne, 1876-1877, p. 6.
} 
Longines ${ }^{29}$. Issue d'un comptoir horloger fondé en 1832 et repris vingt ans plus tard par Ernest Francillon, cette société entreprend, à la fin des années 1860, un processus de rationalisation qui va en faire l'un des géants de l'industrie horlogère suisse. Pour ce faire, Francillon bâtit en 1867 une nouvelle fabrique et fait appel à l'ingénieur Jacques David pour le seconder dans l'organisation de cette nouvelle manufacture. Peu à peu, au cours des années 1870-1900, les dirigeants des Longines concentrent leur main-d'œuvre au sein de l'entreprise et y introduisent des machines. Le nombre d'employés passe de 170 personnes en 1870 à 850 en 1905, date à laquelle les Longines sont la seconde entreprise horlogère du pays. Quant à la production, elle connait aussi une très forte hausse : la production annuelle passe ainsi de 20000 pièces en 1885 à 93000 en 1901. Cette forte croissance nécessite l'engagement en nombre de chefs d'ateliers et de cadres techniques disposant des connaissances permettant l'essor d'une entreprise industrielle, raison pour laquelle Ernest Francillon et Jacques David soutiennent, dès sa fondation, l'École d'horlogerie de la localité.

La décision de créer une école d'horlogerie est prise en septembre 1865 et l'établissement est ouvert l'année suivante, avec une classe unique comprenant trois élèves, dirigée par un horloger. L'année suivante, deux autres classes sont ouvertes, ce qui porte la durée de l'apprentissage à trois ans dès 1868. Cette durée est jugée particulièrement longue par de nombreux parents et apprentis, puisque ce temps durant lequel le jeune homme ne rapporte pas de salaire est considéré comme perdu. Plusieurs personnes demandent que leurs enfants puissent suivre uniquement certains cours pratiques, sur une durée totale de dix-huit mois, ce qu'accepte le comité en 1869. Dans le même ordre d'idées, le cursus est revu en 1872 : les heures de théorie sont réparties en trois divisions, selon le degré d'instruction des élèves avant leur entrée à l'école ; les bons élèves peuvent ainsi entrer en première année de pratique mais en deuxième, voire en troisième année de théorie, afin de rentabiliser leur formation. Le temps ainsi gagné doit permettre un apprentissage sur des parties spéciales en troisième année. L'École cherche ainsi à répondre aux besoins des fabricants et à la demande des apprentis, qui se rendent de plus en plus nombreux dans l'établissement : ils sont 6 en 1866, 27 en 1870 et 30 en 1875 (fig. 1).

29 Sur la fabrique des Longines, voir Patrick Linder, op. cit., et Laurence Marti, Une région au rythme du temps : histoire socio-économique du Vallon de Saint-Imier et ses environs, 1700-2007, Saint-Imier, Éd. Longines, 2007. 


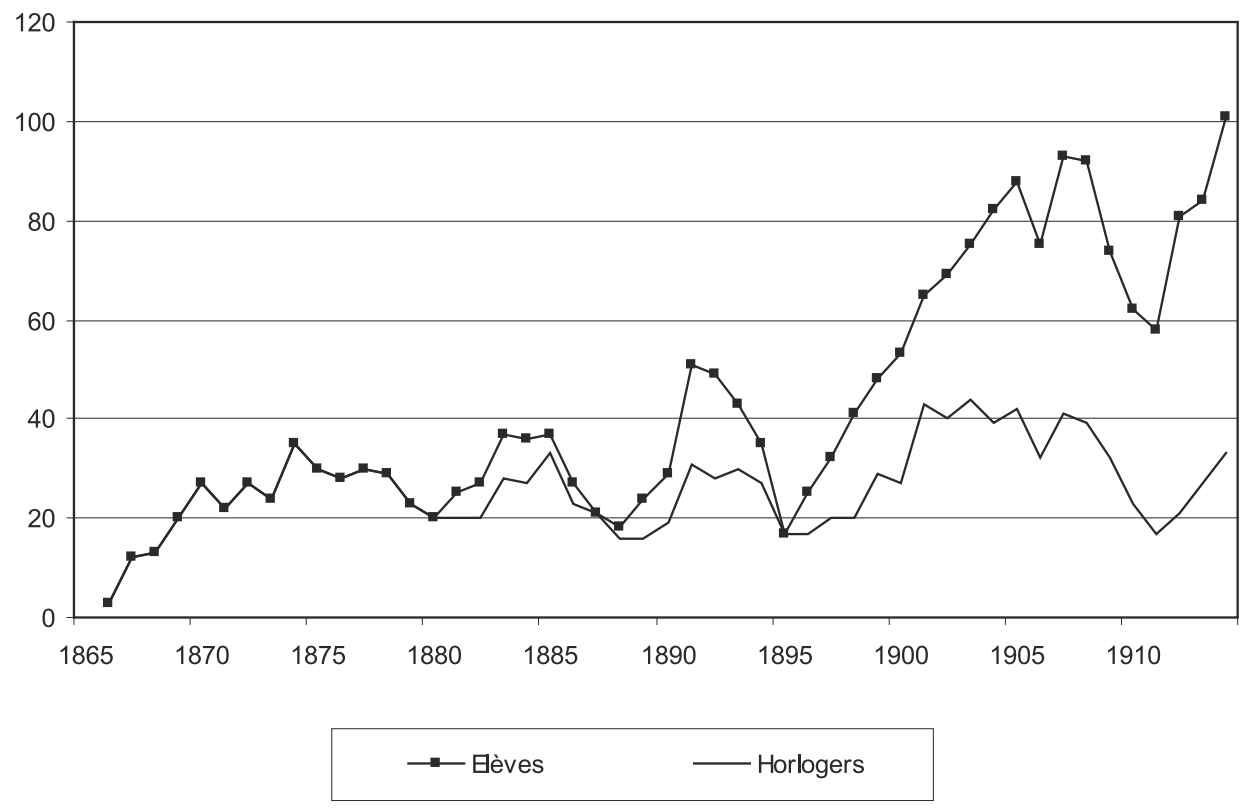

Graphique 1 : Nombre d'élèves de l'École d'horlogerie de Saint-Imier, 1866-1914

Enfin, il faut souligner le soutien précoce de l'État cantonal à cette institution, puisque celui-ci lui verse un subside annuel dès l'année scolaire 1866-1867. Cette aide publique est destinée à " répandre dans notre pays le goût de la fabrication de la belle horlogerie qui est moins sujette que les qualités inférieures aux variations excessives des prix ", ainsi qu'à " élever une génération d'horlogers instruits, habiles à créer des genres nouveaux et à simplifier, au lieu de les copier servilement, les types existants ${ }^{30}$. Mais, dans ses premières années, l'établissement dépend encore très largement des ressources privées, et principalement de l'écolage versé par les élèves. Celui-ci s'élève à 300 francs par an, montant qui peut être réduit pour certains apprentis de familles peu aisées. Pour l'exercice 1872-1873, le total des écolages versés se monte à 4580 francs, soit 51,9\% des ressources ${ }^{31}$.

30 MDI, circulaire de la direction de l'intérieur du canton de Berne aux fabricants d'horlogerie, 6 juillet 1875 .

31 Archives de l'État de Berne (AEB), BB IV 1121, comptes de l'École d'horlogerie de Saint-Imier, 1873. 


\section{1 - Les réformes du directeur Heinis dans les années 1870}

Bien que l'établissement connaisse des succès, à en juger par l'augmentation du nombre de ses apprentis, son organisation interne et son programme de formation sont revus au milieu des années 1870. Ancien maître à l'École d'horlogerie de Besançon, Heinis est nommé directeur en 1872. Son projet de modernisation prévoit aussi bien le renforcement de l'enseignement théorique que le développement de la pratique. Au niveau de la théorie, il demande la création d'une bibliothèque, l'augmentation du nombre de leçons d'arithmétique et de français, ainsi que l'introduction de leçons de cosmographie. Quant à l'enseignement pratique, il est réorganisé en 1874 avec l'introduction d'un nouveau système de formation en lien avec les besoins de l'industrie locale. Le directeur se procure du travail auprès des comptoirs de Saint-Imier et le fait réaliser par les élèves. Ce mode de fonctionnement motive les apprentis par le petit salaire qu'il leur procure et donne une bonne réputation à l'établissement parmi les fabricants, satisfaits de l'aspect utilitariste de l'apprentissage. En effet, selon un rapport de la commission, le nouveau système d'enseignement pratique consiste "à fournir des ouvrages en assez grande quantité pour qu'on puisse adopter le système de travail en parties brisées, tel qu'il est adopté dans les ateliers et reconnu pratique par tous les producteurs "32. Plus les élèves avancent dans l'apprentissage, plus ils font une partie compliquée du processus de fabrication. Cette nouvelle organisation du travail permet à l'École de produire davantage de montres, et de meilleure qualité.

Il ne faut toutefois pas idéaliser les réformes adoptées par Heinis. L'atelier d'horlogerie pose un certain nombre de problèmes d'organisation. La commission reproche au directeur de mener, contrairement à ce que stipule son contrat, une activité de fabricant indépendant depuis 1874 et de négliger ainsi son enseignement, notamment au niveau théorique. Plusieurs apprentis quittent d'ailleurs l'école pour aller terminer leur apprentissage chez un particulier ${ }^{33}$, et le nombre d'élèves est en baisse dans la seconde partie des années 1870, sur fond de crise économique. En 1877, Heinis quitte son poste et est remplacé par de nouveaux directeurs qui continuent à moderniser l'enseignement dispensé dans un sens utilitariste.

32 AEB, BB IV 1121, rapport du 5 avril 1875.

33 AEB, BB IV 1121, lettre de la commission à la Direction de l'Intérieur, n.d. [1876]. 


\section{2 - Le triomphe de la mécanique}

La modernisation de la formation des horlogers s'opère à divers niveaux après 1877. Tout d'abord, il s'agit de renouveler l'équipement et l'outillage utilisés par les apprentis. L'École acquiert ainsi deux tours en 1879 et reçoit, la même année, un premier don de Francillon " pour être employé spécialement au perfectionnement de l'outillage $»^{34}$. Les achats de machines deviennent réguliers au cours des années 1880. Ainsi que la commission l'affirme dans son rapport d'activités de 1885-1886, les deux nouvelles machines achetées durant cette année scolaire sont " destinées comme les précédentes à faire connaitre aux élèves les moyens de production employés dans les fabriques d'horlogerie ${ }^{35}$. Ensuite, on assiste à une ouverture plus large de l'École, dans le sens d'une démocratisation du recrutement, grâce à la baisse de l'écolage (1885). Enfin, l'École diffuse des connaissances relatives à l'horlogerie américaine, qui constitue une référence en matière de production mécanisée. En 1887, la commission demande à la Société intercantonale des industries du Jura " de bien vouloir distribuer sa collection de mouvements américains aux écoles d'horlogerie ; placés dans les collections, ces mouvements rendraient encore des services " ${ }^{36}$. Trois ans plus tard, l'École achète de nouveaux mouvements américains.

À l'intérieur de l'École, la modernisation est prise en charge par un nouveau directeur, James Reymond, nommé en 1880 . Venu de la fabrique d'horlogerie de Montilier, l'une des entreprises horlogères les plus modernes de Suisse, il reste à la tête de l'établissement jusqu'en 1916. Cette modernisation des programmes d'enseignement n'a toutefois pas d'effets chiffrés en termes de recrutement. Pour les années 1880-1900, le nombre d'apprentis horlogers se monte en moyenne à 23,2 élèves par an. Cette relative stagnation est due à la capacité d'accueil limitée de l'établissement. En effet, après l'ouverture d'un nouveau bâtiment, en 1901, on observe une hausse d'une dizaine d'unités du nombre d'apprentis horlogers : leur nombre s'élève à 35,9 par an en moyenne pour les années 1901-1918.

Le programme de formation sur trois ans ne suscite toutefois pas un intérêt généralisé de la part des fabricants. Certains restent fortement attachés au modèle utilitariste et interviennent à nouveau en 1881 pour demander que l'on rétablisse des apprentissages pratiques de 18 mois. La commission accepte cette proposition et une classe pratique d'échappements est ouverte l'année suivante. Ainsi que 
l'explique la commission, cette nouvelle formation fait suite au " changement qui s'est produit ces dernières années dans le système de fabrication de l'horlogerie par la création de fabriques de montres occupant de nombreux ouvriers à des parties brisées (courtes parties de la fabrication) " ${ }^{37}$. Cette nouvelle filière, pour laquelle une seconde classe pratique est créée en 1891, connaît un vif succès. Pour la période 1881-1916, on dénombre en moyenne 12,6 élèves par an dans ces classes, soit $21 \%$ de l'ensemble des élèves de l'école. De plus, sur pression de la chambre de commerce locale, l'écolage pour la classe pratique d'échappements est baissé à 5 francs par mois en 1890, ce qui permet aux enfants des classes peu aisées d'y accéder.

L'introduction de machines qui s'opère au cours des années 1880 et 1890 dans les entreprises horlogères, principalement chez Longines, crée de nouveaux besoins en main-d'œuvre. Il devient, en effet, essentiel de disposer d'un personnel capable de comprendre, de mettre en œuvre et de contrôler l'introduction des machines dans le processus de fabrication des montres. La mécanisation de l'horlogerie nécessite la formation de nouveaux apprentis : les mécaniciens. Une première classe de mécanique est ouverte en 1896. Selon le rapport de l'ingénieur Labhardt, de Saint-Imier, cette école de mécanique " est destinée à devenir une pépinière de bons ouvriers et de contremaitres mécaniciens dont le besoin se fait sentir actuellement " ${ }^{38}$. L'enseignement est axé sur la formation pratique des élèves, en lien avec les besoins de l'industrie horlogère. Il s'agit alors d'apprendre à produire des machines qui serviront à la fabrication de montres. Ainsi, en 1912, le directeur Reymond met au point une nouvelle montre en collaboration avec la classe de mécanique. À l'occasion d'une séance de la commission, il " présente dans deux cartons des ébauches et un mouvement du nouveau calibre établi par lui. L'outillage et la mise en train de ce beau mouvement ont été faits par la classe de mécanique "39. De même, en 1916, la maison Cortébert Watch Co commande à l'École deux types nouveaux de machines à tailler, avec "l'intention de reproduire cette machine dans [son] atelier de mécanique à plusieurs exemplaires ${ }^{40}$. La même année, l'École vend une machine à la maison Berna Watch Co. La filière des mécaniciens est sans conteste celle qui connaît le plus de succès durant la première partie du $\mathrm{XX}^{\mathrm{e}}$ siècle, le nombre d'apprentis dans cette branche

37 AEB, BB IV 1121, lettre de la commission à la Direction de l'Intérieur, 23 mai 1882.

38 AEB, rapport d'activité, 1897-1898, p. 11.

39 MDI, procès-verbaux de la commission, 25 mars 1912.

40 MDI, procès-verbaux de la commission sous-commission mécanique, 21 février 1916. 
étant en constante augmentation jusqu'au début des années 1920 : tandis que l'on compte déjà 17 apprentis mécaniciens en 1900, ils sont 25 en 1910, et 35 en 1920. Sur l'ensemble de la période 1896-1920, les mécaniciens représentent $31,7 \%$ des apprentis.

Enfin, une quatrième filière est ouverte en 1912 avec la création d'une classe de régleuses. Ainsi que l'explique la commission, " sur une demande présentée par les fabricants d'horlogerie de la contrée ", celle-ci décide, entre autres, " d'ouvrir au printemps un cours de réglages et d'admettre des jeunes filles [...]. L'introduction de ce cours dans notre École est une conséquence de la diminution du travail à domicile, du développement de la fabrication mécanique de la montre et du manque d'ouvriers dans cette partie importante de l'horlogerie " ${ }^{41}$. La classe de régleuses prévoit deux types d'apprentissage, de 12 et de 18 mois. Elle comprend une dizaine de jeunes filles (11,6 \% des élèves de l'école entre 1912 et 1918).

\begin{tabular}{|l|c|c|}
\hline & Nombre & En \% \\
\hline Horloger complet (trois ans) & 492 & 50.5 \\
\hline Cours pratiques d'horlogerie (moins de trois ans) & 284 & 29.1 \\
\hline Régleuse (un an et demi) & 32 & 3.3 \\
\hline Mécanicien (trois ans) & 167 & 17.1 \\
\hline Total & 975 & 100 \\
\hline
\end{tabular}

Formation suivie par les élèves de l'École d'horlogerie et de mécanique de Saint-Imier, 1866-1916 (Cinquantenaire de l'École d'horlogerie de Saint-Imier, Saint-Imier, 1916, p. 25.)

Le redéploiement de l'École d'horlogerie observé dans les années 1880-1918 est aussi rendu possible par une modernisation de l'infrastructure et des finances de l'institution. La croissance de l'établissement en termes d'effectifs nécessite la construction d'un nouveau bâtiment. Celle-ci est largement soutenue par les industriels de la ville, tels Ernest Francillon, qui fait un don de 15000 francs pour la constitution d'un fonds de construction pour une nouvelle école (1890), et Georges Agassiz, qui offre le terrain (1893). Le nouvel édifice est ouvert en février 1901. Il restera, jusqu'en 1961, le lieu dans lequel se formera la main-d'œuvre pour l'industrie locale. Quant au fonctionnement financier de l'école, il s'affranchit peu à peu de la dépendance envers les écolages versés par les apprentis, qui représentaient plus de la moitié des ressources au début des années 1870. Ce mode de 
fonctionnement est apparemment inconciliable avec la volonté d'ouverture et de croissance de l'école. Même si les fabricants d'horlogerie, principalement Ernest Francillon, sont les principaux bénéficiaires de la main-d'œuvre ainsi formée, leur participation financière reste limitée. C'est plutôt du côté des collectivités publiques que l'on va chercher des subsides en hausse. Ainsi, en 1880, la part de l'écolage est tombée à 25,3 \% des recettes, tandis que celle du canton de Berne s'élève à $53,6 \%{ }^{42}$. La tendance se renforce encore durant la décennie suivante : en 1890 , les écolages représentent $13 \%$ des recettes, tandis que les collectivités publiques (commune, canton et Confédération) contribuent à hauteur de 63,4 \%.

On a donc affaire dans les années 1880-1920 à une école extrêmement dynamique, qui s'adapte aux différents besoins en main-d'œuvre des entreprises de la région et qui développe ainsi sa capacité de formation. De manière générale, le nombre des élèves est en très forte hausse : il passe de 20 en 1880 à 53 en 1900 et 104 en 1920. Toutefois, ce ne sont plus uniquement des horlogers complets, maitrisant l'art de la montre dans toute sa complexité, qui sortent de l'établissement, mais une main-d'œuvre diverse aux savoir-faire élargis. Au décès de Jacques David, en 1912, l'établissement de Saint-Imier est une école d'horlogerie d'avant-garde, qui non seulement s'est développée en répondant aux besoins en main-d'œuvre d'une région horlogère, mais aussi s'est imposée comme lieu de transmission de nouveaux savoir-faire et de modernisation d'une culture technique régionale.

\section{III - La diffusion du modèle de Saint-Imier en Suisse (1880-1914)}

L'école d'horlogerie telle qu'elle a été restructurée à Saint-Imier dans les années 1880 et 1890 rencontre rapidement un certain succès auprès des autres établissements, qui s'étaient montrés réticents à une transformation en profondeur de leur rôle en 1876-1877. Caractérisé par la prédominance d'un enseignement pratique et proche des besoins de l'industrie, le modèle de Saint-Imier s'impose à l'ensemble des écoles d'horlogerie de Suisse. Toutefois, les modalités de modernisation de la formation scolaire varient dans l'espace. Les établissements de Bienne, de La Chaux-de-Fonds et de Porrentruy illustrent trois types de trajectoires empruntées par les écoles suisses sur la voie de la modernisation. 


\section{1 - Une difficile modernisation : l'École d'horlogerie de Bienne}

La position des dirigeants de l'École d'horlogerie de Bienne face à la question de la modernisation de l'enseignement dans les années 1876-1877 révèle le caractère artisanal de l'établissement. Malgré une première phase de modernisation dans les années 1880, l'école de Bienne poursuit dans la voie de l'excellence technique jusque dans les années 1920.

Dans un premier temps, la commission administrative se montre sensible aux besoins nouveaux des industriels horlogers. Elle met ainsi sur pied, en 1880, des cours pratiques de réglage d'une durée de deux à trois semaines, afin de former rapidement la main-d'œuvre dont les fabriques de la ville ont besoin. Mais surtout, elle décide d'introduire un enseignement de mécanique dans la formation des horlogers. Un nouvel atelier est établi en 1881 et les élèves doivent y passer plusieurs semaines au début de leur apprentissage pour y être " exercés au limage et au tournage de pièces relativement grandes, avant de passer aux délicats travaux de l'horlogerie " ${ }^{43}$. Une formation de mécanicien est même autonomisée dans la seconde partie des années 1880 : cette classe comprend 5 apprentis en 1888 et 10 en 1889 .

Toutefois, la création d'un Technicum cantonal en 1890 vient rompre cette dynamique modernisatrice. Cette nouvelle institution, directement dépendante de l'État, fusionne une dizaine d'écoles techniques et professionnelles (écoles d'horlogerie, de mécanique, des chemins de fer, des postes, d'architecture, etc.) en une seule entité scolaire. Elle a pour conséquence une séparation de la formation des mécaniciens et des horlogers en deux filières distinctes. Tandis que l'École de mécanique modernise rapidement la formation offerte pour répondre aux besoins changeants des entreprises, avec notamment l'introduction de l'électrotechnique dans le cursus, l'École d'horlogerie s'établit dans une poursuite de l'excellence technique qui l'éloigne peu à peu des besoins des entreprises. Bien que cette dernière continue à se développer en ouvrant des classes d'enseignement pratique de courte durée (classe d'horlogers-rhabilleurs ${ }^{44}$ en 1895, classe de régleuses en 1913) et connaisse une hausse du nombre de ses élèves (25,4 apprentis par an, en moyenne, de 1890 à 1899 et 42,5 de 1900 à 1910), elle continue de privilégier un enseignement théorique basé sur l'excellence, dont le but est la formation de techniciens. Les grandes entreprises industrielles de la ville se désintéressent de l'École, à l'exemple de la maison Omega, troisième plus grande fabrique d'hor-

Rapport d'activité, 1881-1882, p. 7.

Rhabillage : action de réparer et de remettre en état de fonctionner une montre. 
logerie de Suisse, qui emploie en 1905 plus de 700 ouvriers dans ses usines ${ }^{45}$. Contrairement à la maison Longines, elle n'intervient pas dans l'École d'horlogerie, ni par des aides financières, ni par une présence au sein de la commission administrative, pour une bonne raison : elle possède son propre centre de formation professionnelle.

Toutes les fabriques industrielles de la ville n'ont toutefois pas les moyens financiers d'Omega et on observe, au début des années 1920, une intervention de la Société des fabricants d'horlogerie de Bienne, qui veut favoriser le développement de l'École en lui donnant une plus grande autonomie. En effet, son intégration dans un Technicum regroupant des filières très différentes pose un certain nombre de problèmes. Selon l'inspecteur fédéral Rosat, qui appuie les fabricants biennois, l'École d'horlogerie " manque d'autonomie relative, c'est-à-dire qu'elle dépend trop d'organes dont il n'est pas possible d'exiger une compréhension parfaite des besoins et des buts de cette institution " ${ }^{46}$. La Société des fabricants revendique une modernisation de l'enseignement, parce qu'" il est dans le rôle de l'École d'horlogerie de répondre aux demandes des fabriques, de peupler ces dernières d'ouvriers et d'ouvrières qualifiés ${ }^{47}$. Elle déplore, en effet, que les apprentis formés à Omega aient "souvent plus de connaissances pratiques que les élèves de l'École d'horlogerie " $"$. Cette intervention porte ses fruits et la direction du Technicum réorganise l'École d'horlogerie au cours des années 1920, avec la construction de nouveaux bâtiments (1923-1925), l'ouverture d'une nouvelle classe de régleuses (1924) et la nomination d'un directeur issu de l'industrie en la personne d'Albert Berner, ancien chef de fabrication de la maison Zénith (1927). Cette restructuration porte ses fruits et permet une forte hausse du nombre d'apprentis (47 en 1920 et 91 en 1930).

\section{2 - Une modernisation réussie : La Chaux-de-Fonds}

L'École d'horlogerie de La Chaux-de-Fonds, telle que mise sur pied au milieu des années 1860, ne donne pas satisfaction à la masse des fabricants de la ville, qui dénoncent rapidement le caractère élitiste de l'établissement. La commission de commerce de la ville écrit ainsi vers 1878 au conseil municipal pour lui

45 Marius Fallet-Scheurer, Le travail à domicile..., op. cit., p. 314.

46 Archives de l'Association cantonale bernoise des fabricants d'horlogerie, procès-verbal d'une séance entre la Société des fabricants d'horlogerie de Bienne et la sous-commission de l'École d'horlogerie, 26 février 1921.

47 Ibid.

48 Ibid. 
faire part de ses désirs relatifs à cette École. Déclarant qu'" il faut en nos temps non seulement de bons horlogers, mais il faut des mécaniciens horlogers " ${ }^{49}$, les membres de cette commission revendiquent une modernisation de l'enseignement dispensé : "Quand l'on voit le développement de la fabrication d'horlogerie américaine, et en considérant les progrès de la science de la mécanique appliquée à l'horlogerie, l'on peut se demander à juste titre si nos Écoles d'horlogerie créées dans les années prospères répondent aujourd'hui encore aux exigences du jour " 50 . Ils demandent que l'École d'horlogerie de La Chaux-de-Fonds s'inspire de celle de Saint-Imier.

Sensibles à cette argumentation, les autorités communales prennent rapidement des mesures afin de transformer l'établissement. En 1879, le montant de l'écolage est abaissé de 25 à 15 francs annuels, pour permettre à un nombre plus grand de jeunes gens de fréquenter les cours ${ }^{51}$. Le nombre d'élèves, qui se montait à 23,1 en moyenne pour la période 1870-1878, va ainsi croitre régulièrement et passer à 65 en 1890 et 77 en 1900. L'instruction des élèves passe aussi par la visite de fabriques d'horlogerie qui travaillent selon un mode de production industrialisé. Ainsi, en 1882, la sortie annuelle emmène les élèves à la fabrique d'horlogerie de Montilier, à propos de laquelle ils doivent rédiger un rapport " sur ce qu'ils auraient pu voir d'intéressant et de nouveau pour eux "52, puis dans trois fabriques de Bienne, dont celle de la maison Omega. De plus, l'école est dirigée depuis 1880 par des hommes favorables à la modernisation de l'horlogerie. Charles Junod, directeur de 1880 à 1883, fait l'acquisition de machines-outils pour les ateliers des apprentis. Il quitte toutefois rapidement l'école pour reprendre la direction technique d'une fabrique d'ébauches et est remplacé en 1884 par un homme qui allie excellence technique et formation mécanique, Paul Berner. C'est sous sa direction que le recours à la machine est intégré dans le cursus de formation. Une classe de mécanique est ouverte en 1886. Très axée sur l'enseignement pratique, elle réalise notamment divers travaux pour des ateliers d'horlogerie de la ville à la fin des années $1880^{53}$. Cette ouverture est suivie en 1889 par celle d'une classe de fabrication mécanique des ébauches. Dès le milieu des années 1880, l'École d'horlogerie offre donc deux types de formations (horloger et mécanicien), répondant

49 ACHX, lettre-circulaire de la commission de commerce, s.d. [1878 ?].

50 Ibid.

51 ACHX, copie du rapport adressé par la commission d'École d'horlogerie au Conseil d'État, 26 février 1880.

52 ACHX, rapport annuel pour 1882.

53 ACHX, L3a11-1889, classe de mécanique, cahier des débiteurs 
ainsi aux besoins variés des fabricants de la ville. Cependant, les deux métiers évoluent selon des trajectoires indépendantes l'une de l'autre, ce qui mène à la fermeture de la classe de fabrication mécanique des ébauches et à la création d'une École de mécanique autonome, qui reste orientée vers les besoins des fabriques d'horlogerie de la localité (1903). Quant à la formation des horlogers proprement dite, elle se caractérise par la mise sur pied d'une filière de techniciens destinée à la formation d'une nouvelle génération de chefs d'ateliers et de directeurs techniques acquis aux nouveautés de la mécanique, dont les premiers diplômés sortent en 1899. Enfin, pour répondre aux besoins des négociants en horlogerie, on ouvre en 1894 une classe de rhabilleurs, dans laquelle sont formés les négociants suisses qui vont s'établir à l'étranger ainsi que de jeunes étrangers embauchés par ces négociants. Cette filière a surtout pour but de transmettre les qualifications nécessaires à l'entretien et à la réparation des montres. Ainsi, les deux dernières décennies du XIX ${ }^{e}$ siècle voient s'autonomiser des métiers spécifiques (mécanicien, horloger, technicien, rhabilleur) pour répondre aux besoins des fabricants de la ville. Le processus est similaire à celui observé à Bienne, mais il se réalise plus précocement et plus rapidement.

\section{3 - La création de nouvelles écoles : le cas de Porrentruy}

Enfin, il faut souligner l'existence de plusieurs écoles d'horlogerie créées après les débats de 1877 sur la modernisation de la formation des horlogers. C'est le cas des établissements de Porrentruy (1882), de Soleure (1884) et de la Vallée de Joux $(1901)^{54}$. Fondées sous l'impulsion des industriels horlogers, ces écoles ont d'emblée une fonction utilitariste : elles ne sont pas créées dans le but de sauvegarder une culture technique que l'on dit menacée, mais pour fournir une maind'œuvre à l'industrie locale. L'École d'horlogerie de Porrentruy est représentative de cette trajectoire ${ }^{55}$.

Fondée en 1882 par des fabricants d'horlogerie de la ville de Porrentruy, l'École de la localité ouvre deux ans plus tard avec 10 élèves. D’emblée, la poursuite d'objectifs pratiques est le but de la commission, qui déclare en mai 1886 vouloir former des apprentis " autant en vue de la célérité dans l'ouvrage, en général, qu'au point de vue de l'exactitude dans l'exécution ${ }^{56}$. Le nombre d'heures de théorie

54 Chantal Schindler-Pittet, "La création de l'École d'horlogerie de la Vallée de Joux ", Revue vaudoise d'histoire, 1976, p. 163-185.

55 Pierre-Yves Donzé, Formation professionnelle et développement industriel dans le district de Porrentruy aux $19^{e}$ et $20^{e}$ siècles, Neuchâtel, Alphil, 2005.

Rapport annuel, 1886-1887, p. 4. 
est réduit au minimum : en 1887, les apprentis travaillent 57 heures par semaine à l'école, dont seulement 9 consacrées à la théorie ${ }^{57}$. Dans la seconde partie des années 1880, l'École fonctionne comme un véritable atelier de sous-traitance pour les fabricants de la ville : la production des élèves leur est vendue, afin d'assurer le financement de l'établissement, et doit donc être rentable. L'inspecteur fédéral Tissot soutient lui-même cette organisation. Lors d'une visite de l'établissement, en 1888, il s'oppose " à ce que l'on fasse beaucoup ou à ce qu'on aille trop loin en théorie, cela charge par trop l'esprit des jeunes gens " ${ }^{58}$. L'orientation pratique de l'enseignement est renforcée en 1888 avec l'ouverture de quatre cours pratiques à durée limitée (plantage d'échappements à ancre, repassage, réglage, remontage) destinés aux ouvriers horlogers de la ville.

Malgré ce succès du principe utilitariste, la formation d'horloger dispensée à Porrentruy n'atteint pas le niveau des autres écoles. La recherche d'une rentabilité à court terme de l'atelier d'apprentis se fait au détriment d'une formation complète et cohérente, qui nécessiterait davantage d'heures de théorie. De plus, passée l'euphorie des débuts, la formation prodiguée à l'École de Porrentruy n'attire pas les jeunes gens, qui préfèrent s'engager directement au sein des fabriques. L'école perd des élèves - ils ne sont plus que 13 en 1893 - et son président, le fabricant Kenel, se demande " si, au vu de la situation de l'industrie horlogère et de l'école, il y a lieu de conserver celle-ci et de lui consacrer les sommes importantes dépensées jusqu'ici " ${ }^{59}$. Une assemblée des fabricants de la ville a lieu en juillet 1894 et voit intervenir l'un des principaux industriels de la ville, Jules Dubail. Déclarant que " la chose principale dans toutes ces discussions est que l'école actuelle est mal dirigée et que les élèves n'y apprennent rien " ${ }^{60}$, celui-ci reprend la présidence de l'institution et réoriente l'enseignement dans le sens d'une réelle formation professionnelle destinée à une nouvelle main-d'œuvre.

Il installe l'école dans de nouveaux locaux et nomme des enseignants ayant une expérience dans les grandes entreprises (1895). Quant à la formation des élèves proprement dite, toujours prévue sur une durée de trois ans, elle est réorganisée en 1895, avec l'adoption d'un nouveau programme qui stipule que " chaque élève doit faire deux pièces à la main, une à savonnette et une à verre, sur calibre spécial

57 Rapport annuel, 1887-1888, p. 6.

58 Centre professionnel de Porrentruy (CPP), procès-verbaux de la commission de l'École d'horlogerie, 9 juin 1888.

59 Ibid., 27 juin 1894.

60 Ibid., 5 juillet 1894 . 
de l'école (remontoir ancre). Depuis la partie des finissages, et pour chacune des parties suivantes, l'élève s'exerce sur des mouvements de fabrique avant d'exécuter la partie correspondante sur ses mouvements propres, afin que les deux montres, sorties complètement des mains de l'élève, puissent représenter le maximum de bienfacture qu'il est capable de produire à sa sortie de l'école "61. En 1904, l'école fait l'acquisition de tours automatiques afin de former les horlogers à la production mécanique. L'année suivante, la commission fait part de son désir " de voir la création d'une petite fabrication des ébauches par procédés mécaniques, pour former les élèves à ce genre de travail " 62 . Ces décisions répondent directement aux attentes des fabricants de la ville, désireux d'engager un personnel technique qui maitrise la production mécanisée de montres. Aussi, en 1907, lorsqu'il faut remplacer un maître de pratique démissionnaire, la commission décide d'engager un diplômé d'une école d'horlogerie ayant l'expérience de la production d'ébauches en fabrique. D’autre part, une classe de régleuses est ouverte vers 1910. Enfin, dans le but de permettre aux nombreux horlogers de la ville de bénéficier du savoir-faire de l'école, des cours du soir sur les échappements - domaine particulièrement compliqué - sont organisés dès l'hiver 1898-1899 à la demande du fabricant Albert Amuat, membre de la commission. Réunissant une quarantaine d'ouvriers de la ville la première année et poursuivis durant les années suivantes, ils sont destinés à diffuser à large échelle certaines innovations techniques, ainsi que l'explique la commission en 1905 : "Notre industrie locale, dont les produits sont encore, dans leur très grande majorité, les montres à cylindre, fait de grands efforts en vue d'implanter dans notre contrée la montre à ancre. Nos fabricants trouvent difficilement les ouvriers nécessaires à cette fabrication nouvelle pour nous. Aussi le besoin de la création d'une classe telle que celle qui est décrite ci-dessus se fait-il vivement sentir "63.

En termes d'effectifs, la réorganisation des années 1890 est tout à fait positive, puisque l'on passe de 6 apprentis en 1895 à 17 en 1900, et la croissance se poursuit jusque dans les années 1920, avec un record de 58 apprentis en 1920. La bonne marche de l'établissement s'explique par une seule raison : la formation prodiguée correspond parfaitement aux besoins d'une industrie modernisée en expansion. Ainsi que l'explique en 1905 la commission dans son rapport d'activité, "l'école est de plus en plus appréciée et ne peut suffire aux demandes qui

61 Cité dans le Journal suisse d'horlogerie, XXI, 1897, p. 29.

62 Journal suisse d'horlogerie, XXIX, 1905, p. 240.

63 CPP, procès-verbaux de la commission de l'École d'horlogerie, 5 avril 1905. 
lui sont adressées par les fabricants. Tous les élèves sont placés bien avant la fin de leur apprentissage, et avantageusement "64.

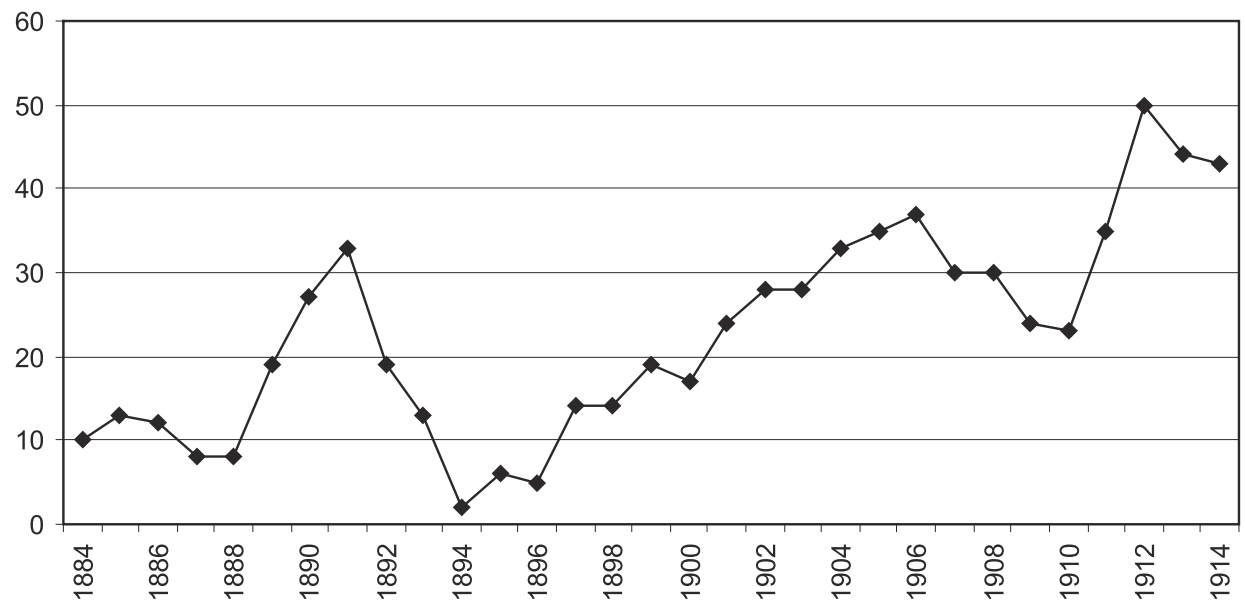

Graphique 2 : Elèves à l'École d'horlogerie de Porrentruy, 1884-1914 (CPP, rapports annuels.)

Les trajectoires suivies par les diverses écoles d'horlogerie de Suisse durant les années 1850-1920 montrent clairement que la culture technique transmise au cours du processus d'apprentissage n'est pas un donné naturel, mais résulte de discussions, de négociations, voire de conflits, au sein de la communauté industrielle à laquelle sont destinés les apprentis de ces établissements. Si, dans un premier temps, les écoles d'horlogerie sont portées par un courant traditionaliste qui cherche à promouvoir une excellence technique, celui-ci n'est plus en phase avec les modes de productions fondés sur une forte division du travail.

La redéfinition de la culture technique transmise dans ces établissements, au cours des années 1870-1900, ne dépend cependant pas uniquement du degré de modernisme du patronat local, mais aussi de l'implication des grands industriels modernisateurs au sein de ces écoles. Certes, une ville comme La Chaux-de-Fonds est connue pour posséder un patronat horloger fort réticent face à la mécanisa- 
tion de ses modes de production dans les dernières décennies du XIX ${ }^{\mathrm{e}}$ siècle $^{65}$, et ce conservatisme technique influence considérablement la voie de l'excellence choisie par l'École d'horlogerie de la ville, abandonnée pourtant dès le début des années 1880. Mais, à l'inverse, il est surprenant d'observer les difficultés avec lesquelles l'école de Bienne entreprend son processus de modernisation, alors même que cette ville est celle dont l'horlogerie connait le plus fort mouvement d'industrialisation dans ces mêmes années. Ce phénomène s'explique par l'absence dans les affaires de l'école du grand patronat modernisateur, en particulier de la famille Brandt, qui possède son propre centre de formation dans ses usines Omega. Ce cas de figure est toutefois exceptionnel et peu représentatif de l'ensemble de l'industrie horlogère. Omega est, avec Longines, la plus grande manufacture de montres du pays en 1914. Toutes deux emploient près d'un millier d'ouvriers dans leurs usines ${ }^{66}$. L'écrasante majorité des fabricants d'horlogerie n'ont toutefois pas les moyens financiers d'Omega. Ils externalisent la formation de leurs employés dans des écoles d'horlogerie fondées collectivement et gérées avec le soutien des collectivités publiques. L'École d'horlogerie de Saint-Imier apparait, dans cette perspective, comme un modèle de modernisme dû à l'implication des dirigeants de la fabrique des Longines, qui en fait sa pépinière d'ouvriers qualifiés ${ }^{67}$. De même, l'École d'horlogerie de Porrentruy, qui emprunte dans les années 1880 une voie extrême vers l'utilitarisme, est dirigée par l'une des seules fabriques industrialisées de la ville.

Malgré les difficultés et les retenues qui s'observent ça et là, on assiste, dès les années 1890-1900, à une certaine unification du modèle institutionnel adopté dans les écoles d'horlogerie suisses. Ayant abandonné l'idéal de l'excellence technique, les directions proposent des établissements relativement ouverts, dans lesquels l'écolage n'est plus un moyen de sélection et dont le but est d'offrir divers types de formation répondant aux besoins variés de l'industrie. D'une culture technique homogène fondée sur l'accumulation de connaissances relevant de

65 Jean-Marc Barrelet, "Les résistances à l'innovation dans l'industrie horlogère des Montagnes neuchâteloises à la fin du XIX siècle ", Revue suisse d'histoire, 1987, p. 394-411, et Pierre-Yves Donzé, Les patrons horlogers de La Chaux-de-Fonds. Dynamique sociale d'une élite industrielle (1840-1920), Neuchâtel, Alphil, sous presse.

66 Hélène Pasquier, La "Recherche et Développement" en horlogerie. Acteurs, stratégies et choix technologiques dans l'arc jurassien suisse (1900-1970), Université de Neuchâtel, thèse de doctorat, 2007, p. 351.

67 Un document établi en 1941 montre qu'à cette date, 186 employés de Longines ont fait leur apprentissage à l'école de Saint-Imier (soit $23 \%$ de l'ensemble du personnel). MDI, Listes des ouvriers et ouvrières ayant fait leur apprentissage à l'École d'horlogerie de Saint-Imier, 1941. 
disciplines différentes, on est passé à une culture technique multiforme et évolutive, caractérisée par son utilitarisme et son adéquation avec le marché. Les écoles d'horlogerie ne servent désormais plus de conservatoires techniques. Elles accompagnent les entreprises dans leur processus de mutation industrielle.

Pierre-Yves DONZÉ

Université de Kyoto, Fonds national suisse py.donze@gmail.com 